\section{Fendas orofaciais no recém-nascido e o uso de medicamentos e condições de saúde materna: estudo caso-controle na cidade do Rio de Janeiro, Brasil}

\author{
Oral clefts in the newborn and medical \\ intakes and maternal health conditions: \\ a case-control study in the city of \\ Rio de Janeiro, Brazil
}

Isabel Cristina G Leite 1

Francisco José Roma Paumgartten 2 Sérgio Koifman 3

1 Universidade Presidente Antônio Carlos. Campus de Juiz de Fora. Av Juiz de Fora, 1100. Granjas Betânia. Juiz de Fora, Minas Gerais, Brasil. CEP: 36.048-000

2 Laboratório de Toxicologia. Fundação Oswaldo Cruz. Rio de Janeiro, RJ, Brasil.

3 Departmento de Epidemiologia e Métodos Quantitativos em Saúde. Escola Nacional de Saúde Pública. Fundação Oswaldo Cruz. Rio de Janeiro, RJ, Brasil.

\begin{abstract}
Objectives: to evaluate the association of this malformation group and specific conditions of maternal health.

Methods: case control study with 274 individuals with cleft lip and palate and 548 hospital controls. The odd ratio $(O R)$ and confidence intervals were obtained based on conditional logistic interval analysis.

Results: the main associations were maternal malformation reports (OR 8,01; 95\%IC\%: 2,2328,81); gynecological infections (OR 2,77; 95\%IC: 1,50-5,10); neurological conditions (OR 7,11; IC95\%: 2,57-19,75); viruses (common cold for example) (OR 7,57; 95\%IC: 2,46-23,32). In reproductive history no condition was demonstrated as being statistically significant, nevertheless, previous spontaneous abortions (OR 1,42; 95\%IC: 0,95-2,13) and stillborns (OR 2,22; 95\%IC: 0,88-5,65) demonstrated more pronounced risks. In addition to the use of anticonvulsant drugs (OR 20,00; 95\%IC: 3,36-437,97), other drug groups were indicated as risk factors such as analgesics (OR 1,45; 95\%IC: 1,08-1,95); antibiotics (OR 2,43; 95\%IC: 1,33-4,57); antifungical drugs (OR 9,00; 95\%IC: 2,14-61,12) and teas (OR 4,09; 95\%IC: 2,237,77).

Conclusions: together with exposures already described in the literature, including the use of anticonvulsant drugs, many others were identified. Although they may have been affected by possible mental confusion and forgetfulness that could have affected the results of this study, they should be evaluated and used as subsidies to more specific studies.
\end{abstract}

Key words Cleft lip, Cleft palate, Pregnancy, Pharmaceutical preparations

\section{Resumo}

Objetivos: avaliar a associação deste grupo de malformações e determinadas condições de saúde materna.

Métodos: estudo caso-controle reunindo 274 portadores de fendas lábio-palatinas e 548 controles hospitalares. As medidas de risco (odds ratio: OR), foram obtidos a partir da análise de regressão logística condicional.

Resultados: as principais associações foram o relato de malformações maternas (OR 8,01; IC95\%: 2,2328,81); infecções ginecológicas (OR 2,77; IC95\%: 1,505,10); distúrbios neurológicos (OR 7,11; IC95\%: 2,5719,75); viroses (resfriados comuns, por exemplo) (OR 7,57; IC95\%: 2,46-23,32). Na história reprodutiva, nenhuma condição foi estatisticamente significantes, no entanto, abortos espontâneos prévios (OR 1,42; IC95\%: 0,95-2,13) e natimortos (OR 2,22; IC95\%: $0,88-5,65)$ exibiram maior magnitude de risco. Além do uso de anticonvulsivantes (OR 20,00; IC95\%: 3,36-437,97), outros grupos de medicamentos atuaram como fatores de risco, como analgésicos (OR 1,45; IC95\%: 1,08-1,95); antibióticos (OR 2,43; IC95\%: 1,33-4,57); antifúngicos (OR 9,00; IC95\%: 2,14-61,12) e chás (OR 4,09; IC95\%: 2,23-7,77).

Conclusões: ao lado de exposições já descritas na literatura, como aquela relativa ao uso de anticonvulsivantes, outras tantas foram identificadas. Embora possam ter sofrido a influência de fatores de confundimento e viés de memória, que podem afetar os resultados deste estudo elas devem ser avaliadas servindo de subsídio para estudos mais específicos. Palavras-chave Fenda labial, Fenda palatina, Gravidez, Preparações farmacêuticas 


\section{Introdução}

Um importante campo de estudos surge no interesse em reconhecer os efeitos da exposição a agentes químicos e potenciais perturbações na reprodução humana e no desenvolvimento fetal. Algumas malformações congênitas estão comprovadamente associadas ao uso de medicamentos em um período gestacional precoce, como é o caso da redução de membros (dentre outros defeitos) causados pelo uso da talidomida e a associação das fendas orofaciais e o uso de drogas anticonvulsivantes.1,2 Contudo, a ação teratogênica de outras drogas mais comumente usadas é controversa. Estatísticas norte-americanas estimam que cerca de metade das gestações não são planejadas $^{3}$ e, portanto, muitas mulheres expõem inadvertidamente seus fetos a ação de medicamentos. Assim, a falta de controle na venda dessas drogas, a prática da automedicação e a recomendação de uso por parte de comerciantes, aliados à falta de programas efetivos de planejamento familiar,contribuem na elevação desse risco. 4

Embora as fendas orofaciais sejam uma das malformações congênitas mais freqüentes e tenham sido descritas pela primeira vez há quase dois séculos, sua etiologia não se encontra claramente estabelecida. Dentre outros fatores ambientais, a exposição a medicamentos parece ter importante ação no mecanismo que interfere no desenvolvimento embriológico, resultando na falha parcial de fusão dos processos nasais médios e outras anormalidades de desenvolvimento.

O objetivo do presente artigo é avaliar se a exposição a diferentes grupos medicamentosos no período de um ano prévio à gestação ou durante seu primeiro trimestre associa-se ao aumento da freqüência de fendas orofaciais típicas. Também serão discutidas condições médicas das mães, representadas por doenças conhecidas e/ou tratadas no mesmo período, bem como sua história reprodutiva.

\section{Métodos}

Os critérios de seleção dos sujeitos e a metodologia aplicada estão descritos em outro estudo. ${ }^{5}$ Os dados foram obtidos a partir de um estudo caso-controle de base hospitalar, conduzido no período de janeiro de 2000 a março de 2001. Os casos foram contactados num hospital pediátrico público no município do Rio de Janeiro, Rio de Janeiro, Brasil. Foram considerados casos os pacientes em primeira consulta e/ou consultas subseqüentes, portadores de fendas orofaciais típicas isoladas, sem indícios de síndromes associadas. Para cada caso foram identificados dois controles, sendo selecionados dentre pacientes internados por diferentes nosologias nas enfermarias do mesmo hospital e em outros hospitais públicos das regiões de procedência dos casos. Foram selecionados como controles crianças com período de internação mínimo de sete dias. Dentre as principais patologias descritas entre os controles, se destacam: complicações de viroses, como varicela; meningite e encefalite; desidratação; doenças ortopédicas; queimaduras; recém-natos em maternidades. Casos e controles foram pareados segundo sexo, idade ( \pm dois meses) e região de procedência, e sua idade não ultrapassou 24 meses. Foram entrevistados 822 responsáveis, sendo 274 casos e 548 controles.

Após aprovação nos comitês de ética institucionais (segundo a resolução $\mathrm{n}^{\circ}$ 196/96 do Conselho Nacional de Saúde), foi feito um estudo piloto e adequação do instrumento de coleta de dados (questionário), bem como a calibração dos entrevistadores. O instrumento de entrevista segue o padrão elaborado e validado pela International Agency for Research on Câncer (IARC) para coleta de dados relativos a questões ambientais e ocupacionais. 6 Foram coletadas variáveis de identificação e socioeconômicas; dados relativos a consagüinidade e história familiar de malformação; hábitos sociais (incluindo o tabagismo passivo, através do pai, fumo no ambiente doméstico ou de trabalho); contaminação ambiental percebida (uso de inseticidas domésticos e/ou agrícolas), presença de indústrias nas circunvizinhanças; história médica e de uso de medicamentos; dieta; histórico ocupacional materno e paterno. A exposição pesquisada referia-se ao período de um ano prévio à gestação e no primeiro trimestre da gravidez em questão.

As medidas de risco, Odds Ratio (OR) e seus respectivos intervalos de confiança de 95\% (IC95\%) foram calculados na análise de regressão condicional utilizando o programa Statistical and Epidemiology Research Corporation (Egret), 1995. A análise estratificada de Mantel-Haentzel foi conduzida em algumas situações para avaliar tendência de risco. Foram conduzidas análises distintas para fendas lábio-palatinas $(\mathrm{FL} \pm \mathrm{P})$ e fendas palatinas isoladas (FP). As variáveis independentes de interesse neste estudo foram aspectos da vida reprodutiva, relato de morbidade e uso de medicamentos (prescritos ou de uso espontâneo). Durante as análises uni e bivariada foram avaliadas possibilidade de confundimento, quando a OR por estrato apresentavam variação superior a $20 \%$ da OR bruta, bem como a interação, através do qui-quadrado de Wolff.7 Nas análises de regressão, foram controladas as variáveis idade 
materna, uma vez que a literatura relata aumento da probabilidade de anomalias congênitas associadas ao aumento da idade e escolaridade, que apesar de não exibir significativas diferenças entre os casos e controles na maioria dos estratos, apresentava diferença no nível médio de escolaridade, (o qual reunia alguns casos de formação na área técnica da saúde). Alguns autores sugerem que ocupações relacionadas à área da saúde exibem comportamento de risco para fendas orofaciais. As OR obtidas foram também ajustadas para o número de gestações prévias ao probando, uma vez que a infertilidade pode ser considerada uma possível variável de confundimento com relação a morbidades ginecológicas e uso de drogas de ação hormonal, bem como a variável morbidade referida (uma vez que foi detectada diferença estatisticamente significativa entre os percentuais de exposição a medicamentos entre mães com/sem relato de doença prévia). A análise estratificada, contudo, não detectou o fenômeno de interação, considerando o potencial de confundimento mínimo. O critério de modelagem adotado foi o de plausibilidade biológica, utilizando o método enter.

\section{Resultados}

\section{$\underline{\text { Perfil geral da população estudada }}$}

Na amostra estudada, a análise das variáveis sóciodemográficas demonstra que quanto à escolaridade materna, não houve diferença estatisticamente significante entre casos e controles, exceto na freqüência de mães com ensino médio completo (19,7\% e $11,8 \%$, respectivamente, com $p<0,003)$. O ensino fundamental incompleto (até quatro anos de escolaridade) foi predominante em ambos grupos $(50,7 \% \mathrm{x}$ $54,8 \%$ ). A razão de sexo (M/F) foi superior a um em todos os tipos de fendas, exceto para as fendas palatinas. A idade média das crianças foi de 231,34 dias (correspondendo a 7,7 meses).

Tabela 1

Perfil geral da população estudada.

\begin{tabular}{|c|c|c|c|c|}
\hline \multirow{2}{*}{ Características } & Controles & $\mathrm{FL} \pm \mathrm{P}$ & FP & {$[(F L \pm P)+F P]$} \\
\hline & $(n=548)$ & $(n=208)$ & $(n=66)$ & $(n=274)$ \\
\hline \multicolumn{5}{|l|}{ (anos completos) } \\
\hline $\begin{array}{l}\text { Média de idade paterna } \\
\text { (anos completos) }\end{array}$ & $28 \pm 8,11$ & $28 \pm 7,88$ & $29 \pm 8,80$ & $28 \pm 8,78$ \\
\hline Idade média do afetado (meses) & $7,8 \pm 7,1$ & $7,9 \pm 7,2$ & $7,2 \pm 6,7$ & $7,7 \pm 7,1$ \\
\hline \multicolumn{5}{|l|}{ Idade gestacional em semanas (\%) } \\
\hline$<37$ semanas & $20,1 \%$ & $23 \%$ & $23,7 \%$ & $29,2 \%$ \\
\hline Entre 37 e 42 semanas & $73,9 \%$ & $68,5 \%$ & $67,2 \%$ & $56,6 \%$ \\
\hline$>42$ semanas & $6,0 \%$ & $8,6 \%$ & $9,1 \%$ & $14,2 \%$ \\
\hline \multicolumn{5}{|l|}{ Peso do afetado ao nascer (g) } \\
\hline Média geral & $3085 \pm 642$ & $3163 \pm 620$ & $3170 \pm 595$ & $3112 \pm 555$ \\
\hline$<37$ semanas & $2683 \pm 720$ & $2722 \pm 715$ & $2565 \pm 729$ & $2908 \pm 500$ \\
\hline Entre 37 e 42 semanas & $3222 \pm 513$ & $3242 \pm 531$ & $3218 \pm 437$ & $3275 \pm 616$ \\
\hline$>42$ semanas & $3293 \pm 529$ & $3389 \pm 529$ & $3004 \pm 560$ & $3261 \pm 453$ \\
\hline \multicolumn{5}{|c|}{ Comprimento do afetado ao nascer $(\mathrm{cm})$} \\
\hline Média geral & $49,2 \pm 4.07$ & $49,3 \pm 3,43$ & $49,1 \pm 4,65$ & $49,3 \pm 3,06$ \\
\hline$<37$ semanas & $47,5 \pm 3,65$ & $47,8 \pm 3,80$ & $46,8 \pm 4,37$ & $47,5 \pm 3,96$ \\
\hline Entre 37 e 42 semanas & $47,5 \pm 3,96$ & $49,6 \pm 3,26$ & $49,2 \pm 4,69$ & $49,5 \pm 2,65$ \\
\hline$>42$ semanas & $50,2 \pm 2,88$ & $50,4 \pm 2,52$ & $49,3 \pm 3,81$ & $49,7 \pm 2,42$ \\
\hline
\end{tabular}

$\mathrm{FL} \pm \mathrm{P}=$ fendas labiais com ou sem envolvimento palatino; $\mathrm{FP}=$ fendas palatinas isoladas; $[(\mathrm{FL} \pm \mathrm{P})+\mathrm{FP}]=$ todos os casos de fendas orofaciais não-sindrômicas 
Foi estatisticamente significante a associação deste grupo de malformações e a história familiar pregressa de fendas orofaciais (OR 14,01; IC95\%: 6,32-32,01 e OR 5,96, IC95\%: 3,03-11,84, respectivamente para relatos de história na família paterna e na materna), bem como o relato de parentesco entre os pais para $\mathrm{FL} \pm \mathrm{P}(\mathrm{OR} 3,80$; IC95\%: 1,27-12,18).

Quanto aos hábitos sociais, o tabagismo materno diferiu entre casos e controles $(\mathrm{p}<0,03)$ no período prévio à gestação. Particularmente para $\mathrm{FL} \pm \mathrm{P}$, uma discreta magnitude de risco foi verificada para a exposição habitual no lar/trabalho (OR 1,39; IC95\%: 1,01-1,98). O tabagismo materno prévio à gestação gerou medida de associação estatisticamente significante, embora de pequena magnitude (OR 1,54; IC95\%: 1,05-2,21). O etilismo esteve associado aos desfechos em questão (OR 2,04; IC95\%: 1,33-3,13 para FL \pm P; OR 2,57; IC95\%: 1,27-5,59 para FP) tanto no período prévio quanto no primeiro trimestre da gestação (OR 2,08; IC95\%: 1,27-3,41 para FL \pm P; OR 2,89; IC95\%: 1,25-8,30 para FP). 5

\section{Aspectos relacionados à saúde materna e uso de medicamentos}

Foram analisados 274 grupamentos (casos/ controles), sendo que na Tabela 2 são apresentadas as medidas de risco geradas pelo relato de doenças sistêmicas identificadas e/ou tratadas na mãe. Foram observadas elevadas magnitudes de risco para o histórico de malformação congênita (OR 8,01; IC95\%: 2,23-28,81), infecções ginecológicas (OR 2,77; IC95\%: 1,50-5,10), disritmias/ convulsões (OR 7,11; IC95\%: 2,57-19,75), viroses, incluindo resfriados recorrentes, hepatite (OR 7,57; IC95\%: $2,46-23,32)$ e outras doenças, dentre as quais doenças pulmonares obstrutivas crônicas (OR 2,04; IC95\%: 1,18-3,51). Essa tendência, vista no total de casos, é reproduzida entre as $\mathrm{FL} \pm \mathrm{P}$. Entre as $\mathrm{FP}$, não foi possível a construção de medidas de risco, ou, uma vez obtidas, geraram intervalos de confiança muito amplos, não significantes. Quanto ao grupo de malformações maternas, $4,4 \%$ do total de mães de casos exibiam alguma malformação, sendo que dos 12 casos, $75 \%$ eram fendas orofaciais, $16,6 \%$ polidactilia e $8,4 \%$ outras formas de anomalias congênitas.

\section{Tabela 2}

Situação de saúde materna (ano prévio até $1^{\circ}$ trimestre de gestação), segundo morbidade referida.

\begin{tabular}{|c|c|c|c|c|c|c|}
\hline \multirow{2}{*}{ Doença identificada/tratada } & \multirow{2}{*}{$\mathrm{FL} \pm \mathrm{P}$} & \multirow{2}{*}{$F P(n f)$} & \multirow{2}{*}{ Controles } & \multirow{2}{*}{$\frac{\mathbf{F L} \pm \mathbf{P}}{\mathrm{OR}^{\mathrm{a}}(\mathrm{IC} 95 \%)}$} & \multirow{2}{*}{$\begin{array}{c}\text { FP } \\
\text { OR }(I C 95 \%)\end{array}$} & \multirow{2}{*}{$\frac{[(F L \pm P)+F P]}{O R(I C 95 \%)}$} \\
\hline & & & & & & \\
\hline Não & 119 & 44 & 422 & $1,00 \quad(----)$ & $1,00(---)$ & $1,00(---)$ \\
\hline Sim & 89 & 22 & 129 & $2,41 \quad(1,71-3,55)$ & $1,77(0,87-3,57)$ & $2,25(1,63-3,10)$ \\
\hline Hipertensão & 23 & 9 & 45 & $1,48(0,82-2,02)$ & $1,55 \quad(0,61-3,79)$ & $1,49 \quad(0,89-2,47)$ \\
\hline Malformação & 11 & 1 & 3 & $10,50(2,54-68,65)$ & ---- & $8,01 \quad(2,23-28,81)$ \\
\hline Doenças sexualmente transmissíveisb & 23 & 2 & 26 & $3,67 \quad(1,80-7,54)$ & $0,67 \quad(0,09-3,15)$ & $2,77(1,50-5,10)$ \\
\hline AIDS & 3 & 6 & 24 & $0,56(0,21-1,44)$ & $1,20 \quad(0,40-3,50)$ & $0,80 \quad(0,38-1,66)$ \\
\hline Distúrbios neurológicos c & 12 & 4 & 5 & $4,80(1,73-15,15)$ & --- & $7,11 \quad(2,57-19,75)$ \\
\hline Diabetes & 2 & 4 & 3 & $2,00(0,21-19,21)$ & $8,45 \quad(0,85-206,30)$ & $4,09(0,89-21,02)$ \\
\hline Viroses d & 11 & 3 & 4 & $7,40(2,03-26,95)$ & $6,00 \quad(0,64-157,98)$ & $7,57 \quad(2,46-23,32)$ \\
\hline Outras e & 24 & 5 & 29 & $2,54(1,36-4,74)$ & $1,11 \quad(0,34-3,32)$ & $2,04 \quad(1,18-3,51)$ \\
\hline
\end{tabular}

$F L \pm P=$ fendas labiais com ou sem envolvimento palatino; $F P=$ fendas palatinas isoladas; $[(F L \pm P)+F P]=$ todos os casos de fendas orofaciais não- sindrômicas; a ajustado por escolaridade, idade materna e gestações prévias; $b$ reunindo condiloma acuminado, sífilis, gonorréia; c incluindo alterações encefalográficas e quadros convulsivos; d resfriados comuns e doenças infecto-contagiosas de curta duração; e reunindo doenças respiratórias obstrutivas crônicas, especialmente bronquite; f excluídos indeterminados. 
Analisando a história reprodutiva deste grupo de mães, identifica-se que o relato de abortos espontâneos ou natimortos foi mais comum entre casos, gerando medidas de risco de pequena magnitude, sem poder estatístico. (Tabela 3)

Na gravidez índice, o relato de tentativa de aborto não diferiu entre casos e controles, sendo respectivamente $25,2 \%$ e $28,1 \%(p=0,33)$. Entre os casos, as drogas mais comumente citadas para fins abortivos foram chás de plantas da família Lauraceae (chá de canela), que em altas doses provocam irritação das mucosas e hematúria, e da família Curcubiaceae (chá de buchinha), que, dentre suas aplicações, destaca-se o tratamento da amenorréia. Os relatos incluíam seu uso isolado ou combinado a bebidas não alcoólicas a base de cola e cafeína; uso de drogas de ação analgésica e antipirética, como dipirona sódica, ácido acetilsalicílico associado a paracetamol e cafeína; drogas de ação uterotônica, como a metilergometrina, dentre outros métodos menos freqüentes. Foi também comum o uso de medicamento à base de misoprostol, com a indicação terapêutica em caso de úlceras gástricas, apresentando, entretanto, ação abortiva já identificada, levando a contrações uterinas e expulsão do feto. 8

Tabela 3

Características maternas quanto ao histórico reprodutivo prévio à gestação índice.

\begin{tabular}{|c|c|c|c|c|c|c|}
\hline \multirow{2}{*}{ Dimensão do riscoa } & \multirow{2}{*}{$\mathrm{FL} \pm \mathrm{P}$} & \multirow{2}{*}{ FP $(n *)$} & \multirow{2}{*}{ Controles } & $\mathrm{FL} \pm \mathrm{P}$ & FP & {$[(F L \pm P)+F P]$} \\
\hline & & & & OR $($ IC95\%) & OR (IC95\%) & OR $(I C 95 \%)$ \\
\hline História prévia de aborto provocado & 26 & 7 & 57 & $1,42(0,66-2,28)$ & $1,41(0,66-3,11)$ & $1,22(0,75-1,93)$ \\
\hline Nascidos vivos & 117 & 30 & 304 & $1,06(0,76-1,48)$ & $0,56(0,29-1,06)$ & $1,10(0,82-1,47)$ \\
\hline Nascimentos múlitplos & 3 & --- & 12 & $0,63(0,13-2,42)$ & ---- & $0,50(0,11-1,85)$ \\
\hline Gravidez ectópica & 1 & --- & 1 & $1,00(0,02-48,31)$ & --- & $0,67(0,02-9,05)$ \\
\hline História prévia de aborto espontâneo & 34 & 14 & 72 & $1,42(0,81-2,02)$ & $1,41(0,66-3,11)$ & $1,42(0,95-2,13)$ \\
\hline Natimortos & 6 & 4 & 9 & $2,00(0,61-6,55)$ & $2,67(0,55-14,29)$ & $2,22(0,88-5,65)$ \\
\hline
\end{tabular}

$F L \pm P=$ fendas labiais com ou sem envolvimento palatino; $F P=$ fendas palatinas isoladas; $[(F L \pm P)+F P]=$ todos os casos de fendas orofaciais não-sindrômicas; * excluídos registros indeterminados; a OR calculada tendo como referência a ausência do fator de risco.

O relato de um início de gravidez conturbado, com um freqüente estado de tensão/nervosismo (processo de separação, morte de parente/amigo, violência local e familiar, por exemplo) foi mais comum entre casos $(80,0 \%$ e $72,0 \%$, respectivamente entre casos e controles) gerando risco de 1,79 (IC95\%: 1,22-2,94).

$\mathrm{O}$ uso de medicamentos nos dois períodos estudados pode ser analisado na Tabela 4. Não foi discriminado o uso recomendado por um profissional de saúde ou a auto-medicação. Foram incluídos no grupo outros medicamentos drogas de ação contra-abortiva não hormonal e broncodilatores, principalmente. Dentre os chás caseiros mais relatados, foram citados freqüentemente o chá preto, mate, a canela, a cidreira e o limão. Particularmente para $\mathrm{FL} \pm \mathrm{P}$, geraram medidas de risco significantes estatisticamente o grupo de antibióticos $(\mathrm{OR}=3,16$; IC95\%: 1,49-6,70), anticonvulsivantes (OR $=14,00$; IC95\%: 1,80-630,97); antieméticos $(\mathrm{OR}=2,96$; IC95\%: 1,74-4,93) e o grupo de medicamentos diversos (broncodilatadores como fenotenol, salbutamol) com OR =3,50 (IC95\%: 1,27-10,14). A análise estratificada por relato de doença identificada/tratada (morbidade referida pela mãe) exibiu associação com o relato de uso de medicamentos. Assim, mães que relatavam doença prévia exibiam maiores percentuais de exposição a drogas medicamentosas. No entanto, análise estratificada não identificou interação, considerando mínimo o potencial de confundimento exercido por essa variável na associação entre o uso de medicamentos e o desfecho em estudo. 
Odds ratio ajustada para o uso de medicamentos no período prévio à gestação e no primeiro trimestre e a ocorrência de fendas orofaciais.

\begin{tabular}{|c|c|c|c|c|c|c|}
\hline \multirow{2}{*}{ Grupo de medicamentos } & \multirow{2}{*}{$\mathrm{FL} \pm \mathrm{P}$} & \multirow{2}{*}{$\operatorname{FP}(n *)$} & \multirow{2}{*}{ Controles } & $\mathrm{FL} \pm \mathrm{P}$ & FP & {$[(F L \pm P)+F P]$} \\
\hline & & & & $O R * *(I C 95 \%)$ & OR (IC95\%) & OR (IC95\%) \\
\hline \multicolumn{7}{|l|}{ Analgésico } \\
\hline Uso no ano prévio à gestação Índice & 159 & 53 & 372 & $1,66(1,08-2,47)$ & $2,54(1,16-5,52)$ & $1,63(1,16-2,29)$ \\
\hline Uso no primeiro trimestre & 108 & 31 & 229 & $1,49(1,06-2,15)$ & $1,36(0,75-2,47)$ & $1,45(1,08-1,95)$ \\
\hline \multicolumn{7}{|l|}{ Antibiótico } \\
\hline Uso no ano prévio à gestação índice & 26 & 8 & 46 & $1,70(0,98-2,97)$ & $1,08(0,39-2,88)$ & $1,49(0,92-2,38)$ \\
\hline Uso no primeiro trimestre & 19 & 7 & 19 & $3,16(1,49-6,70)$ & $2,00(0,61-6,55)$ & $2,47(1,33-4,57)$ \\
\hline \multicolumn{7}{|l|}{ Anticonvulsivante } \\
\hline Uso no ano prévio à gestação índice & 10 & 5 & 1 & $20,00(3,36-437,87)$ & --- & $30,00(5,36-637,79)$ \\
\hline Uso no primeiro trimestre & 7 & 3 & 1 & $14,00(1,80-630,97)$ & --- & $20,00(3,36-437,87)$ \\
\hline \multicolumn{7}{|l|}{ Antiemético } \\
\hline Uso no ano prévio à gestação índice & 9 & 2 & 2 & $18,00(2,49-788,96)$ & $4,00(0,30-117,99)$ & $11,52(2,52-52,83)$ \\
\hline Uso no primeiro trimestre & 42 & 18 & 45 & $2,96(1,74-4,93)$ & $4,43(1,78-11,17)$ & $1,16(0,22-27,92)$ \\
\hline \multicolumn{7}{|l|}{ Anti-hipertensivo } \\
\hline Uso no ano prévio à gestação índice & 3 & 4 & 8 & $1,00(0,20-4,02)$ & $4,00(0,71-31,22)$ & $1,75(0,60-4,97)$ \\
\hline Uso no primeiro trimestre & 6 & 4 & 10 & $1,53(0,46-4,96)$ & $4,00(0,30-31,22)$ & $2,09(0,82--5,35)$ \\
\hline \multicolumn{7}{|l|}{ Vitaminas } \\
\hline Uso no ano prévio à gestação índice & 12 & 6 & 24 & $1,33(0,62-2,84)$ & $2,00(0,61-6,55)$ & $1,46(0,77-2,78)$ \\
\hline Uso no primeiro trimestre & 125 & 33 & 295 & $0,87(0,64-1,89)$ & $0,89(0,50-1,59)$ & $1,12(0,84-1,51)$ \\
\hline \multicolumn{7}{|l|}{ Antiinflamatório } \\
\hline Uso no ano prévio à gestação índice & 22 & 9 & 37 & $1,82(0,97-3,28)$ & $2,14(0,73-5,97)$ & $1,85(1,10-3,12)$ \\
\hline Uso no primeiro trimestre & 14 & 2 & 5 & $7,00(2,41-24,71)$ & $4,00(0,30-117,99)$ & $6,45(2,43-19,53)$ \\
\hline \multicolumn{7}{|l|}{ Hipnótico } \\
\hline Uso no ano prévio à gestação índice & 8 & 2 & 6 & $5,84(1,52-22,38)$ & $1,72(0,26-11,23)$ & $3,49(1,25-9,77)$ \\
\hline Uso no primeiro trimestre & 4 & 1 & 4 & $2,88(0,63-13,02)$ & $2,56(0,15-42,34)$ & $2,58(0,69-9,74)$ \\
\hline \multicolumn{7}{|l|}{ Hormônio } \\
\hline Uso no ano prévio à gestação índice & 5 & 3 & 2 & $9,00(1,15-208,03)$ & --- & $14,55(2,29-327,95)$ \\
\hline Uso no primeiro trimestre & 2 & 1 & 3 & $3,00(0,18-88,16)$ & $2,00(0,05-78,00)$ & $2,38(0,33-20,95)$ \\
\hline \multicolumn{7}{|l|}{ Anticoncepcionais orais/ injetáveis } \\
\hline Uso no ano prévio à gestação índice & 108 & 34 & 241 & $1,42(1,02-1,95)$ & $1,22(0,68-2,29)$ & $1,37(1,03-1,83)$ \\
\hline Uso no primeiro trimestre & 30 & 17 & 87 & $0,94(0,58-1,52)$ & $1,56(0,76-3,21)$ & $1,10(0,74-1,64)$ \\
\hline \multicolumn{7}{|l|}{ Outros } \\
\hline Uso no ano prévio à gestação índice & 8 & 1 & 9 & $3,08(1,89-5,50)$ & $0,50(0,02-3,98)$ & $2,00(0,77-5,19)$ \\
\hline Uso no primeiro trimestre & 11 & 2 & 11 & $3,50(1,27-10,14)$ & $1,33(0,16-8,97)$ & $2,75(1,16-6,73)$ \\
\hline \multicolumn{7}{|l|}{ Chás } \\
\hline Uso no ano prévio à gestação índice & 35 & 14 & 25 & $4,64(2,47-8,80)$ & $6,50(2,41-34,86)$ & $5,16(2,99-9,24)$ \\
\hline Uso no primeiro trimestre & 23 & 9 & 17 & $3,75(1,86-7,75)$ & $5,54(1,55-25,58)$ & $4,09(2,23-7,77)$ \\
\hline
\end{tabular}

$\mathrm{FL} \pm \mathrm{P}=$ fendas labiais com ou sem envolvimento palatino; $\mathrm{FP}=$ fendas palatinas isoladas; $[(\mathrm{FL} \pm \mathrm{P})+\mathrm{FP}]=$ todos os casos de fendas orofaciais não- sindrômicas; * excluídas respostas ignoradas; OR calculada tendo como referência a ausência do fator de risco; ** ajustado por escolaridade, idade materna, gestações prévias, uso de outros medicamentos e morbidade referida. 


\section{Discussão}

Os resultados deste caso-controle hospitalar demonstram elevação do risco de nascimento de crianças portadoras de fendas orofaciais típicas de mães com história de malformação; infecções ginecológicas; epilepsia/convulsões; viroses e outras doenças. Alguns desses achados corroboram outros estudos. 9,10 Enfermidades crônicas que afetam as mães, mesmo antes da concepção, podem afetar também seus conceptos, por meio de alterações de sua fisiologia ou pela ação de drogas usadas em seu tratamento. $4 \mathrm{~A}$ associação de história de malformação orofacial da mãe corrobora a literatura vigente na qual a hereditariedade desempenha um papel preponderante na etiologia desse distúrbio específico, o que não é tão evidente entre casos de FP. No que diz respeito às enfermidades contagiosas, na amostra estudada destacaram-se as viroses (agrupando especialmente resfriados comuns, dois casos de citomegalovirose e um de hepatite A); infecções ginecológicas, incluindo candidíase, sífilis, tricomoníase, dentre outras, excetuando AIDS); condições médicas diversas, reunindo principalmente doenças pulmonares crônicas obstrutivas. Essa tendência é também demonstrada no grupo de $\mathrm{FL} \pm \mathrm{P}$. Poucos estudos têm enfatizado a ampla informação quanto às condições sistêmicas de interesse médico, priorizando o uso de medicamentos, 11,12 embora a não aderência a tratamentos seja comum por diversas motivos. A literatura reporta a hipertemia materna como um potencial teratógeno com danos ao desenvolvimento do SNC e malformações faciais, incluindo fendas orofaciais. Alguns estudos in vitro demonstram que a elevação de 1,5 a $2,5^{\circ} \mathrm{C}$ acima da temperatura corpórea normal representa um risco à organogênese, sendo que tanto pequenas, porém freqüentes, elevações da temperatura são tão deletérias quanto picos de grande elevação. ${ }^{13}$ A elevação da temperatura reduz a atividade mitótica de células e pode levar à morte celular por meio da produção de grupos de proteínas (heat shock ou stress proteins) que também são induzidas pela hipóxia e pelo etanol. Em uma amostra independente de casos de FL \pm P houve associação entre essa malformação e o relato de quadros de resfriados e períodos febris ao longo do primeiro trimestre de gestação. 14

Particularmente no caso de $\mathrm{FL} \pm \mathrm{P}$, a condição materna mais freqüentemente associada é a epilepsia, embora vários autores tenham observado que a elevação do risco ocorre somente entre as crianças cujas mães tenham sido submetidas à terapia com drogas antiepiléticas, particularmente a politerapia. $1,4,10$ Neste estudo, foram detectados 21 relatos de epilepsia/convulsões, sendo que das nove mães com relato de uso freqüente de terapia antiepilética (especialmente fenobarbital) 100\% tiveram conceptos portadores da malformação em questão. De 12 casos de uso eventual/isolado, sete apresentavam $\mathrm{FL} \pm \mathrm{P}$.

Analisando o uso de medicamentos, chama a atenção as medidas de risco geradas pela exposição ao grupo dos antibióticos (penicilinas biossintéticas, ampicilinas e tetraciclinas), antifúngicos (nistatina, especialmente), antiinflamatórios (diclofenacos sódico e potássico), grupos de drogas diversas (broncodilatadores como fenotenol, salbutamol). Um estudo nacional 9 também observou o uso de antiinflamatórios com fator de risco (OR 2,54; IC95\%: $1,35-4,98)$ durante os quatro primeiros meses de gestação, ao passo que os achados de um estudo inglês 1 não sustentou essas observações (OR 1,7; IC95\%: 0,6-4,5).

$\mathrm{Na}$ amostra estudada não foi constatado o uso de qualquer antiinflamatório de ação hormonal (como beclometasona, betametasona, dexametasona, hidrocortisona) dentre os controles, não permitindo, dessa forma, estimar medidas de risco. Alguns autores 11 associam o uso de corticosteróides a ocorrência de fendas orofaciais. Outro estudo,12 conduzindo a análise usando controles pareados, gerou estimativa de risco de 5,00 (IC95\%: 0,57-112,6). Usando controles malformados, reduziu o efeito, porém alcançou significância estatística (OR 3,2; IC95\%: 1,07-8,69). Portanto, o uso de corticóides parece ter um efeito nocivo no desenvolvimento do complexo orofacial.

O uso de antieméticos esteve associado à ocorrência de $\mathrm{FL} \pm \mathrm{P}$. Esse achado contraria outros estudos. 1,9

Os grupos de drogas de ação hormonal (anticoncepcionais orais/injetáveis) mais relatados nesse casocontrole foram o etinilestradiol, enantato de estradiol, noretisterona, acetato de ciproterona e acetofenido de algestona. $\mathrm{O}$ uso desses medicamentos gerou medidas de risco significativas para o conjunto de malformações e $\mathrm{FL} \pm \mathrm{P}$ no ano prévio à gestação. Entretanto, em nenhum grupo esse mesmo comportamento foi detectado durante o primeiro trimestre. Esses achados corroboram achados de outra análise ${ }^{1}$ que não alcançou nenhuma medida de associação estatisticamente significativa, tanto para o conjunto de hormônios esteróides analisados, quanto para contraceptivos orais exclusivos ou hormônios progestacionais e para aqueles usados no tratamento de infertilidade. Da mesma forma, a exposição pré-natal a progesterona e outras combinações excluindo estrogênios também exibiu comportamento de risco. 15 
Os dados do presente estudo indicam um consumo freqüente de chás no período prévio e no primeiro trimestre da gestação em questão (49/274 casos e 25/551 controles no período prévio e 32/274 casos e 17/551 controles no primeiro trimestre). Esse consumo gerou em todos os grupos $[(\mathrm{FL} \pm \mathrm{P})+\mathrm{FP}]$ estimativas de risco estatisticamente significantes. Nenhum relato semelhante foi descrito na literatura para $\mathrm{FL} \pm$ P. Paralelamente, o efeito nocivo da exposição à cafeína é controverso na literatura, na qual apesar das evidências de sua não teratogenicidade relatada em alguns estudos, 16 outros autores associam seu elevado consumo pré e perigestacional à elevação do risco de abortos espontâneos, aberrações cromossômicas, múltiplas anomalias congênitas 17 e especialmente baixo peso ao nascer. 18

O relato do uso de vitaminas sugeriu uma ação de proteção contra $\mathrm{FL} \pm \mathrm{P}$ e FP durante o primeiro trimestre, embora não estatisticamente significantes. No grupo analisado, a suplementação polivitamínica ou associação de vitaminas do complexo B foram os mais freqüentemente citados, sendo poucos os relatos de uso exclusivo de ácido fólico, habitualmente, feitos por mães com história de malformação própria ou em gestação prévia. Uma análise sobre suplementação multivitamínica entre mães de crianças portadoras de diversas malformações, comparou o relato de mães de controles divididos em dois grupos: saudáveis e malformados (outras categorias). ${ }^{19}$ A análise foi referente a cada mês do primeiro trimestre, bem como no mês prévio à gestação (28 dias antes do último ciclo menstrual). A comparação tanto de controles saudáveis quanto malformados revelou a exposição em questão como fator de proteção tanto para grupos com $\mathrm{FL} \pm \mathrm{P}$ quanto FP, com medidas estatisticamente significantes para FP no primeiro mês da gestação (controles saudáveis: OR 0,1; IC95\%: 0,04-0,4; controles malformados: OR 0,2; IC95\%: 0,1-0,6). Parece ainda ser obscuro qual grupo (ou grupos) vitamínico(s) tem efeito protetor contra o desenvolvimento de fendas orofaciais. ${ }^{20}$ Além de um possível efeito direto na prevenção de malformações, dentre outros, nota-se que pacientes subnutridas têm maiores riscos de infertilidade, retardo no crescimento, abortos, nascimentos prematuros e aumento da mortalidade perinatal.

Uma importante questão metodológica em estudos caso-controle refere-se ao viés de memória.
No caso de relatos assimétricos (erro diferencial), no qual as mães de casos se lembrassem mais freqüentemente do uso de medicamentos, poderiam ser geradas estimativas de risco espúrias, ou no caso de erro não diferencial a tendência é a redução do poder explicativo. Uma alternativa para minimizar essa possibilidade é a utilização de questionários que associem o uso de medicamentos com a sua indicação. Essa observação é feita por autores ${ }^{19}$ que aplicaram uma medida de estimativa do viés de memória (sensibilidade relativa), resultante da razão da acurácia das resposta sobre exposições perigestacionais entre mães de casos e controles. Os autores observaram que situações de tratamento contra infecções urinárias, história de infertilidade e medicações de ação hormonal após a concepção exibiram as maiores medidas, caracterizando erro diferencial. Para minimizar esses efeitos, os autores sugerem também a utilização de controles malformados. Essa seleção de controles é, no entanto, criticada por outros autores, 21 que admitem que o uso de controles não saudáveis poderia ser útil para mensurar a especificidade da associação entre a exposição e um particular defeito, mas não permitiria a estimativa de uma razão de risco propriamente dita. O presente estudo buscou minimizar essa possibilidade utilizando-se de controles hospitalares, não portadores de malformação, com período de internação de, pelo menos, sete dias, bem como a limitação da idade de casos e controles de, no máximo, 24 meses e a utilização de questionários com a indicação do uso de medicamentos. Por outro lado, dada as circunstâncias de coleta de dados, não era possível manter o entrevistador "cego" para a condição da criança selecionada para entrevista.

Os dados do presente estudo indicam um potencial efeito teratogênico no uso de certas drogas, como antibióticos e antiinflamatórios. Não é reproduzida, por outro lado, a associação entre o uso de drogas de ação hormonal e a ocorrência deste grupo de malformação. A consistência das medidas de risco alcançadas e a descrições similares na literatura suportam estes achados, bem como o rigor na seleção do grupo controle, representado por pacientes hospitalizados, sugere uma redução particular do viés de memória diferencial, embora especialmente em exposições pontuais, como no uso de medicamentos eventuais, sua influência na obtenção das medidas de risco não possa ser completamente descartada. 


\section{Agradecimentos}

À direção do Hospital Municipal Nossa Senhora do Loreto, na pessoa de seu diretor na ocasião do estudo, Dr. Samuel Warth, ao Centro de Estudos do referido hospital, presidido pela fonoaudióloga $\mathrm{Dr}^{\mathrm{a}}$. Cynthia Maria de Biasi Britto, ao setor de Fonoaudiologia e sua coordenadora Dr ${ }^{\mathrm{a}}$ Maria Célia Rendeiro, à área de Odontologia sob responsabilidade da Dra. Maria de Fátima Brandão, bem como a todo o staff do Centro de Tratamento de Fissuras Lábio-palatais (CEFIL) coordenado pelo Dr. Sérgio Zanini. Apoio financeiro: CNPq (processo 142027/00-6) e Fundação Oswaldo Cruz (FIOCRUZ).

\section{Referências}

1. Hill L, Murphy M, McdowalL M, Paul AH. Maternal drug histories and congenital malformations: limb reduction defects and oral clefts. J Epidemiol Comm Health 1988; 42: 1-7.

2. Mc Bride WG. Thalidomide and congenital abnormalities. Lancet 1961; 2: 1358.

3. Dolovich LR, Addis A, Vaillancourt JMR, Power JDB, Koren G, Einarson TR. Benzodiazepine use in pregnancy and major malformations or oral clefts: meta-analysis of cohort and case-control studies. Br Med J 1998; 317: 839-43.

4. Castilla E, Camelo-Lopez JS, Paz JÁ, Orioli IM. Prevención primaria de los defectos congénitos. Rio de Janeiro: FIOCRUZ; 1996.

5. Leite ICG, Paumgartten JFR, Koifman S. Orofacial clefts in the newborn and environmental and occupational parental exposures: a case-control study in Rio de Janeiro, Brazil. Rev Bras Saúde Matern Infant 2003; 3: 401-9.

6. Boffetta P, Brennann P, Hererro R. Occupational questionnaire. Research protocol: Viruses, environment and cancer of the oral cavity and the larynx in Mercosul Countries". France: Lyon International Agency of Research on Cancer and World Health Organization; 1998.

7. Lilienfield DE, Stolley PD. Foudations of epidemiology. Oxford: Oxford University Press; 1994. p. 123-5.

8. Ellertson C, Waldman SN. The mifepristone-misoprostol regimen for early medical abortion. Curr Women Health Reprod 2001; 1: 184-90.

9. Loffredo LC, Souza JM, Yunes J, Freitas JA, Spiri WC. Cleft lip and palate: case-control study. Rev Saúde Pública 1994; 28: 213-7.

10. Wyszynski DF, Beaty TH. Review of the role of potential teratogens in the origin of human nonsyndromic oral clefts. Teratology 1996; 53: 309-17.
11. Carmichael SL, Shaw GM. Maternal corticosteroid use and risk of selected congenital anomalies. Am J Med Gen 1999; 86: $242-4$.

12. Rodríguez-Pinilla E, Martínez-Frías ML. Corticosteroids during pregnancy and oral clefts: a case-control study. Teratology 1998; 58: 2-5.

13. Germain MA, Webster WS, Edwards MJ. Hyperthermia as a teratogen: a parameters determining hyperthermia-induced head defects in teh rat. Teratology 1985; 31: 265-72.

14. Saxén I. Epidemiology of cleft lip and palate. An attempt to rule out correlations. Br J Prev Soc Med 1975; 29: 103-10.

15. Martínez-Frías ML, Rodríguez-Pinilla E, Bermejo E, Prieto L. Prenatal exposure to sex hormones: a case-control study. Teratology 1998; 57: 8-12.

16. Tikkanen J, Heinonen OP. Maternal exposure to chemical and physical factors during pregnancy and cardiovascular malformations in offspring. Teratology 1991; 43: 591-600.

17. Cristian MS, Brent RL. Teratogen update: evaluation of the reproductive and developmental risks of caffeine. Teratology 2001; 64: 1-78.

18. Hinds TS, West WL, Knight EM, Harland BF. The effect of caffeine on pregnancy outcome variables. Nutr Rev 1996; 54: 203-7.

19. Werler MM, Hayes C, Louik C, Shapiro S, Mitchell AA. Multivitamin supplementation and risk of birth defects. Am J Epidemiol 1999; 150: 675-82.

20. Zhang J, Cai WW. Association of the common cold in the first trimester of pregnancy with birth defects. Pediatrics 1993; 92: 559-63.

21. Prieto L, Martínez-Frías ML. Case-control studies using only malformed infants: are we interpreting the results correctly? Teratology 1999; 60: 1-2. 